\title{
The Effect of Cholecystokinin-Octapeptide, Insulin, Glucagon, Triiodothyronine, and Epidermal Growth Factor on Amylase Activity in Fetal Pancreas in Vitro ${ }^{1}$
}

\author{
JACQUES SARLES, PING-CHEUNG LEE, AND EMANUEL LEBENTHAL
}

International Institute for Infant Nutrition and Gastrointestinal Disease, Children's Hospital of Buffalo and Department of Pediatrics, State University of New York at Buffalo, Buffalo, New York 14222

\begin{abstract}
To evaluate the possible role of various hormones on fetal pancreas development, late gestational fetal rat pancreata ( 20 days) were cultured in a serum-free medium for 6 days in the presence of cholecystokininoctapeptide (CCK-8), epidermal growth factor, triiodothyronine, or glucagon with or without dexamethasone. In the absence of any added hormone, the tissue amylase activity declined very rapidly. Epidermal growth factor alone (4. $10^{-7} \mathrm{M}$ ) could not preserve the amylase activity, whereas triiodothyronine $(0.1 \mu \mathrm{M})$ and glucagon $(4 \mu \mathrm{g} / \mathrm{ml})$ had a deleterious effect that was prevented by the addition of DXM $\left(3 \cdot 10^{-6} \mathrm{M}\right)$. In the presence of CCK-8 $\left(2 \cdot 10^{-11} \mathrm{M}\right)$ 50 and $30 \%$ of the amylase activity was maintained on the 2nd and the 4th day of culture, respectively. The CCK-8 effect was dose dependent and was inhibited by asperlicin $(10 \mu \mathrm{M})$. The combination of CCK-8 and dexamethasone maintained more than $80 \%$ of the amylase activity in the fetal pancreas explants through 4 days of culture. Fetal pancreas cultured in this optimal medium and treated with streptozotocin $\left(10^{-7} \mathrm{M}\right)$ during the 1 st day of culture showed a significantly lower tissue amylase activity on the 4th and 6th days than those not treated with streptozotocin. The streptozotocin effect was attenuated when insulin (0.1 $\mathrm{U} / \mathrm{ml}$ ) was added. These data suggest that, in addition to the well-known effect of glucocorticoid on enzyme activities in the fetal pancreas, two additional hormones, CCK and insulin, could play a role in the modulation of pancreatic amylase activity in the fetal rat. (Pediatr Res 23: 539-542, 1988
\end{abstract}

\section{Abbreviations}

CCK, cholecystokinin

EGF, epidermal growth factor

DXM, dexamethasone

SFM, serum-free medium

BSA, bovine serum albumin

CCK-8, CCK-octapeptide

T3, triiodothyronine

ASA, amylase-specific activity

Received September 14, 1987; accepted January 20, 1988.

Correspondence Emanuel Lebenthal, M.D., International Institute for Infant Nutrition and Gastrointestinal Disease, Children's Hospital of Buffalo, 219 Bryant Street, Buffalo, NY 14222.

${ }^{1}$ Presented in part in the American Pancreatic Association Meeting in Chicago, November 1987.
In the rat, the development of the exocrine pancreatic enzymes has been shown to be a three-phase process (1). The primary transition (day 10 of gestation) leads to the accumulation of very low specific activities of exocrine enzymes without any zymogen granules. Then, according with the appearance of the granules, the activities of exocrine enzymes start to increase dramatically at day 15 of gestation. Finally, shortly after birth, the third phase leads to a decrease of most of the enzymes. The role of hormones in these perinatal changes was first suggested by the fact that the pancreatic mass of newborn rats was decreased by decapitation in utero (2). In vivo experiments have shown that hydrocortisone and CCK increased the amylase-specific activity in the term fetal pancreas (3-5), whereas thyroxine and glucagon did not modulate this enzyme at the same developmental stage (3). These in vivo studies have several disadvantages, i.e. the effect of the placental barrier and the possible complication of mother-fetus interaction during the experiments. To bypass these pitfalls, several groups developed in vitro models that enabled them to show that corticosteroids (but not gonadal steroids) increased the amount of enzymes in cultured fetal pancreata but did not precociously induce their appearance (6-8). Later in gestation, corticosteroids prevented the decline in amylase and acinar cells that usually occurs in pancreatic rudiments placed in culture at that time $(8,9)$. To our knowledge there is no report on a comprehensive study with other hormones that are known to affect the pancreatic development. The aim of this study was to test the effect on prenatal pancreatic development of hormones that have been proven to modulate the amylase activity in the adult, such as CCK (10) and glucagon (11), or in the developing rat, such as thyroid hormones (12), CCK (13), and EGF (14). Pancreatic rudiments were cultured in a chemically defined medium to test the possible direct effect of these hormones when added singly to the medium. Their ability to modulate the effect of glucocorticoids was also evaluated by monitoring their effects in combination with DMX. Furthermore, despite the fact that the addition of insulin was shown to have no effect on amylase activity in cultured fetal pancreas (15), the role of endogenous insulin was further examined by using streptozotocin to suppress insulin production by the explants.

\section{MATERIALS AND METHODS}

Animals. Sprague-Dawley male and female rats were mated and the next morning the females were checked for the presence of a vaginal plug and for sperm in the vagina. Day 0 of pregnancy was defined as the time when sperm-positive vaginas were detected. The females were isolated during pregnancy and killed by decapitation at day 20 of pregnancy. Uteri were quickly excised 
and fetuses were removed. During the time of fetus dissection, the pancreata were pooled in a Petri dish containing $4 \mathrm{ml}$ of SFM at room temperature. The pancreata were then carefully cut into $0.5 \mathrm{~mm}^{3}$ explants and randomly distributed into different dishes for cultures.

Media. The SFM consisted of $50 \%$ Dulbecco modified Eagle medium (GIBCO, Grand Island, NY) and 50\% RPMI 1640 (GIBCO), with HEPES pH 7.4-25 mM (USBC, Cleveland, OH), penicillin $50 \mathrm{U} / \mathrm{ml}+$ streptomycin $50 \mu \mathrm{g} / \mathrm{ml}$ (GIBCO), and BSA $2 \mathrm{mg} / \mathrm{dl}$ (Sigma Chemical Co., St. Louis, MO). Stock solutions were prepared for additive products, kept at $-20^{\circ} \mathrm{C}$, and used at the following final concentrations: Asperlicin $10 \mu \mathrm{M}$, kindly provided by Dr. R. Chang, Merck \& Co., Inc., West Point, PA; CCK-8 $2 \cdot 10^{-11} \mathrm{M}$, Research Plus, Inc., Bayonne and Dennville, NJ; DXM 3. $10^{-6} \mathrm{M}$, Sigma streptozotocin $10^{-7} \mathrm{M}$, Sigma; and $\mathrm{T}_{3} 0.1 \mu \mathrm{M}$, Sigma. Stock solutions for DXM and asperlicin were prepared in ethanol.

Cultures. Petri dishes containing nine to 12 pancreatic fragments suspended in $3 \mathrm{ml}$ of medium were incubated at $37^{\circ} \mathrm{C}$ in a humid atmosphere of $5 \% \mathrm{CO}_{2}$ in air. During the culture period, the media were chánged daily. Three or four pancreatic fragments were withdrawn at day 2,4 , and 6 of culture. They were sonicated in $100 \mu \mathrm{l}$ of water and stored at $-80^{\circ} \mathrm{C}$. At day 6 of culture, all the samples were thawed and assayed for amylase and protein. When streptozotocin was used, it was present in the medium only on the 1st day of culture.

Assays. Protein was determined by the technique of Lowry et al. (16) using BSA fraction V as the standard. Amylase was measured using the Phadebas amylase test from Pharmacia (Piscataway, NJ).

Calculations. Specific activities were calculated and normalized relative to that found at day 0 of culture which was taken as the $100 \%$ value. Values are reported as means \pm SD with significance determined by the Student's $t$ test (two-tailed).

\section{RESULTS}

Cultures without DXM. When cultured in SFM alone (Fig. $1 A$ ) the pancreatic explants degenerated very quickly. After 2
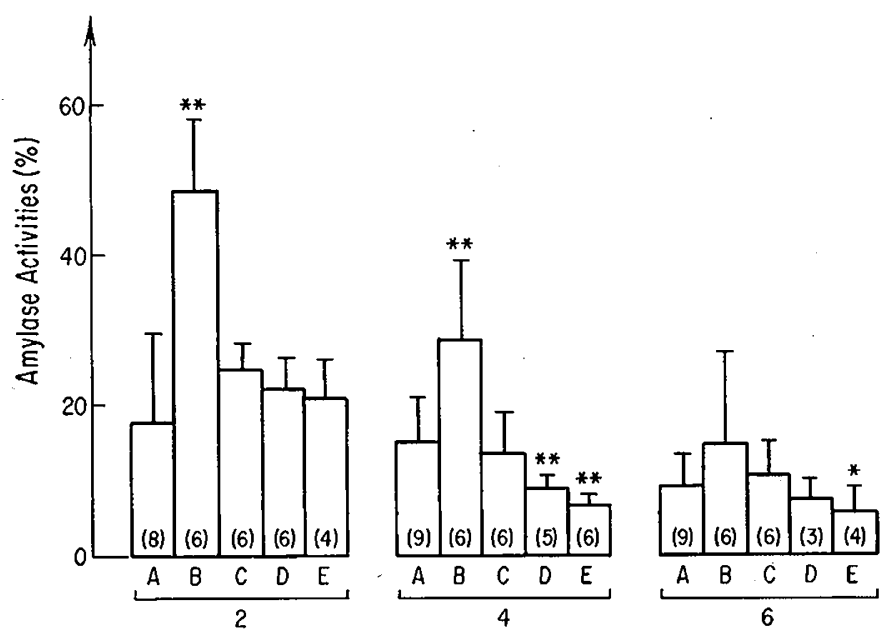

Days of Culture

Fig. 1. Effect of various hormones on amylase activity in fetal pancreas cultured in a SFM. ASA were normalized relative to the values obtained on day 0 for each experiment. Each column represents the percentage [mean \pm SD of $(n)$ experiments] of the activity after 2,4 , and 6 days of culture in the hormone-free medium $(A)$, or in media containing $2 \cdot 10^{-11}$ M CCK- $8(B) ; 4 \cdot 10^{-7}$ M EGF $(C) ; 0.1 \mu \mathrm{M}$ T3 $(D)$; or thyroxin $\mu \mathrm{g} / \mathrm{ml}$ glucagon $(E)$ Asterisks indicate significant differences in comparison to $A ;{ }^{*} p<0.05,{ }^{* *} p<0.01$. days of culture, the ASA was already less than $20 \%$ of the starting value, and further declined thereafter. The addition of CCK-8 to the SFM (Fig. $1 B$ ) allowed a significantly better maintenance of the ASA, at least for the first 4 days of culture. This effect was lost on day 6. In the presence of EGF (Fig 1C), the ASA was not different from that in SFM cultures. $\mathrm{T}_{3}$ and glucagon (Fig. $1 D$ and $E$, respectively), however, had a deleterious effect on the tissue amylase activity after 4 days of culture.

Cultures with $D X M$. In the presence of DXM, the maintenance of the amylase activity was much better than in SFM alone (compare columns $A$ in Figs. 1 and 2). Almost $50 \%$ of the initial ASA was maintained during the first 4 days of culture with glucocorticoid. Ethanol alone, in the same concentration as that used with DXM, had no effect on the cultures (data not shown). The best results were obtained when CCK- 8 was added to the DXM-containing medium (Fig. 2B). CCK-8 and DXM together maintained more than $80 \%$ of the starting ASA during 4 days of culture and it was still more than $60 \%$ on day 6 . In some cultures, this CCK-8-DXM combination induced an increase of ASA on day 2. In contrast, none of the three other hormones tested (EGF, $\mathrm{T}_{3}$, glucagon) significantly change the effect of DXM on the ASA in the cultured fetal pancreata. Glucagon still reduced the tissue amylase activity in the presence of DXM but not significantly.

Dose-response effect of $C C K-8$. Despite the fact that the CCK8 concentration used in the cultures was in a range known to be effective in stimulating adult pancreatic acinar cell growth (17), we did not know if this was also true for the fetal pancreas. The dose-response curve (Fig. 3) showed that the effect of CCK-8 on the maintenance of ASA in fetal pancreas after 2 days of culture was dose-dependent with a maximum occurring between $10^{-12}$ and $10^{-10} \mathrm{M}$.
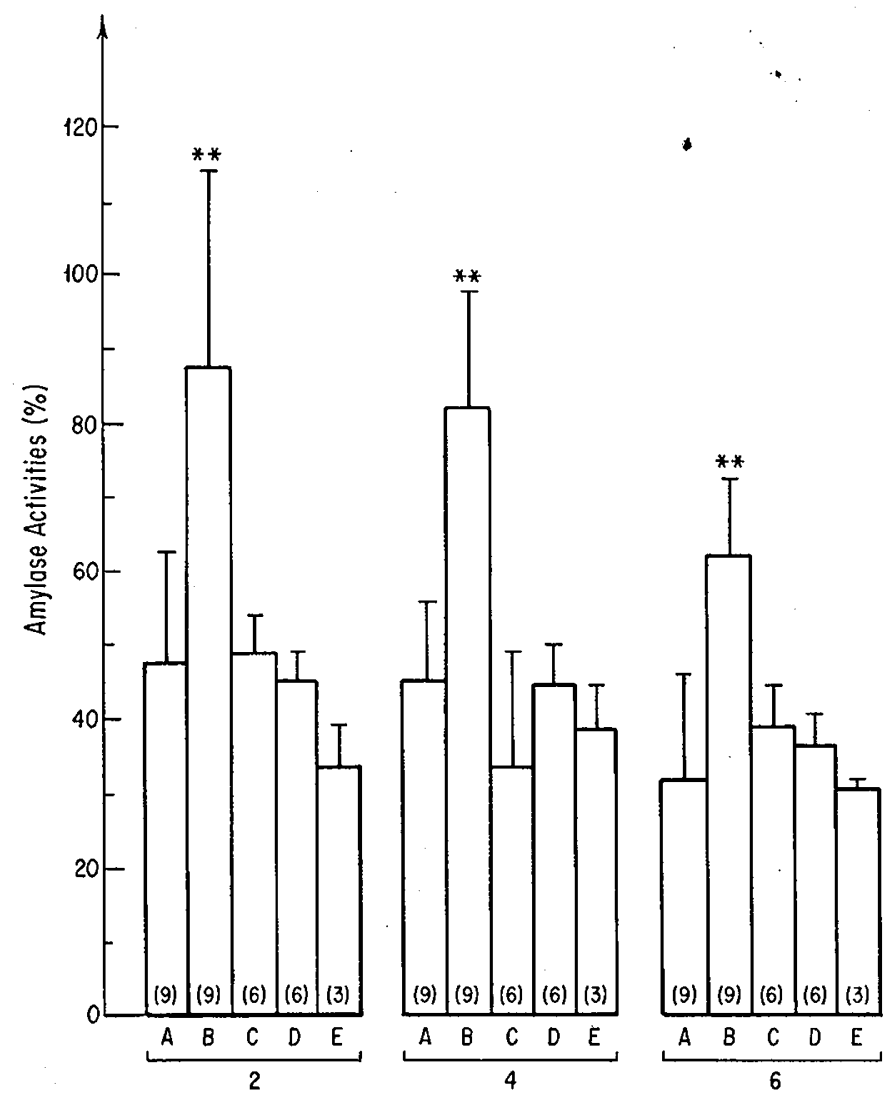

Days of Culture

Fig. 2. Effect of various hormones on amylase activity in fetal pancreas cultured in the presence of DXM. See Figure 1 for explanation except that all the media contain $3 \cdot 10^{-6} \mathrm{M}$ DXM. 


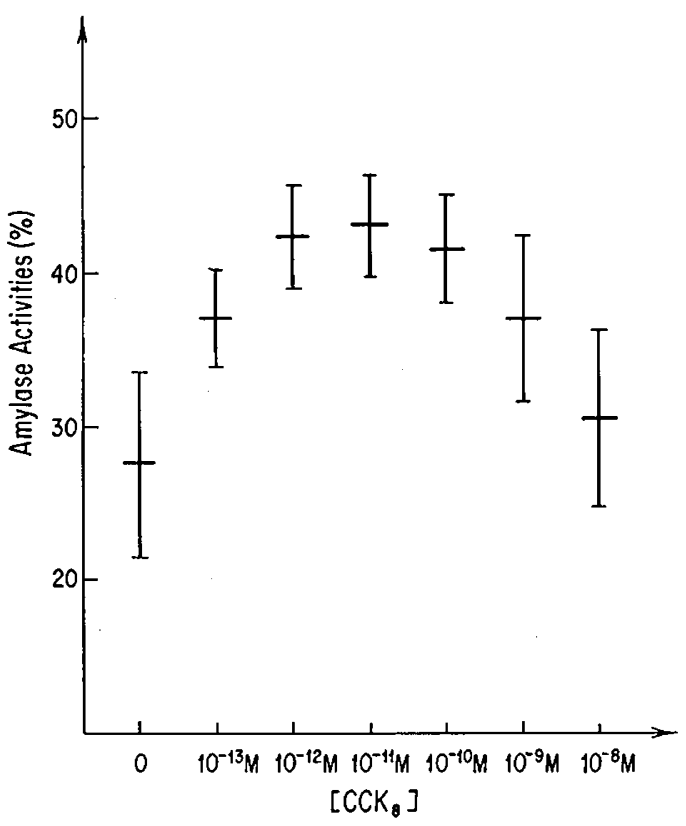

Fig. 3. Dose-response curve of $\mathrm{CCK}$ on amylase activities in fetal pancreas cultured in SFM. ASA was measured on day 2 of culture and normalized as explained in Figure 1. Values represent mean $\pm \mathrm{SD}$ of three experiments.

Inhibition of the $C C K-8$ effect. When asperlicin, a potent $\mathrm{CCK}$ inhibitor (18), was added to the cultures carried out in the optimal medium (containing CCK-8 and DXM), the effect of CCK-8 on ASA was suppressed (Table 1). The enzyme activities were reduced to a level close to that obtained with DXM alone.

Effect of streptozotocin. When cultures (containing CCK-8 and DXM) were exposed to streptozotocin for 1 day, a significant decrease of the ASA was found subsequently on days 4 and 6 of culture, as compared to those levels found in the absence of streptozotocin in the same media (Table 2). This difference was corrected when in the same protocol insulin was present throughout the entire period of culture.

\section{DISCUSSION}

The dramatic rise in the enzymatic activities that occurs on day 15 of gestation in the fetal rat pancreas seems to be mainly genetically determined, as shown by the ability of the pancreas to differentiate in culture (19). Yet exogenous factors, such as hormone, have been shown to play a role in this process (see Ref. 1 for review). Herein, we were able to show that, in addition to the well-known effect of glucocorticoid, other hormones could modulate the enzyme activities in the late fetal exocrine pancreas, at least in vitro.

Inasmuch as EGF has been shown to increase the enzyme activities in the brush-border of the fetal mouse small intestine (20), its possible role on another organ of the digestive tract at the same developmental stage was intriguing. Furthermore, a study in our laboratory showed that the fetal pancreas had a high capacity for EGF binding (21). Nevertheless the physiological relevance of these observations remained uncertain because no significant EGF transfer through the placenta could be demonstrated in the late gestational period in the rat (21). In the current study, EGF had no effect on ASA in cultured fetal rat pancreas with or without DXM (Figs. 1 and $2, C$ ). This fact does not rule out a possible trophic effect of EGF on the fetal pancreas, but points out that if present, it does not act on the exocrine enzyme activities.

Other studies in our laboratory have shown that thyroxin induces a precocious postnatal pancreatic development, in terms of enzyme activities (22), but also has an indirect effect by
Table 1. Inhibition by asperlicin of CCK effect on amylase in fetal pancreas cultured with or without DXM*

\begin{tabular}{llccc}
\hline & \multicolumn{4}{c}{ ASA (\% of day 0) } \\
\cline { 2 - 5 } \multicolumn{1}{c}{ Treatment } & $(n)$ & Day 2 & Day 4 & Day 6 \\
\hline DXM & $(9)$ & $47.9 \pm 14.4 \%$ & $45.3 \pm 0.4 \%$ & $36.5 \pm 9.3 \%$ \\
CCK & $(6)$ & $46.8 \pm 8.8 \%$ & $28.7 \pm 10.8 \%$ & $14.5 \pm 12 \%$ \\
CCK + ASP & $(3)$ & $23.3 \pm 3.7 \% \dagger$ & $19.3 \pm 4.9 \%$ & $14.3 \pm 2.6 \%$ \\
CCK + DXM & $(9)$ & $87.4 \pm 21.8 \%$ & $81.9 \pm 15.9 \%$ & $62.2 \pm 10.3 \%$ \\
CCK + DXM + ASP (3) & $59 \pm 6.1 \% \ddagger 50.6 \pm 9.6 \% \dagger$ & $41.6 \pm 5.3 \% \dagger$ \\
\hline
\end{tabular}

* ASA were normalized as explained in Figure 1. Each value represents the percentage [mean $\pm \operatorname{SD}$ of $(n)$ experiments] of the activity after 2,4 , and 6 days of culture in SFM containing in different combinations 2 . $10^{-11} \mathrm{M}$ CCK-8 (CCK), $10 \mu \mathrm{M}$ asperlicin (AsP), or $3 \cdot 10^{-6} \mathrm{M} \mathrm{DXM}$.

$\dagger \ddagger$ Indicate significant differences between $\mathrm{CCK}+\mathrm{ASP}$ and CCK, or $\mathrm{CCK}+\mathrm{DXM}+\mathrm{ASP}$ and CCK + DXM. $\ddagger p<0.05, \dagger p<0.01$

Table 2. Effect of streptozotocin on amylase activity in cultured fetal pancreas*

\begin{tabular}{llccc}
\hline & & \multicolumn{3}{c}{ ASA (\% of controls) } \\
\cline { 3 - 5 } Treatment & $(n)$ & Day 2 & Day 4 & Day 6 \\
\hline STZ & $(6)$ & $85.2 \pm 10.4 \%$ & $74.6 \pm 21.6 \%$ & $77.1 \pm 8.7 \% \dagger$ \\
STZ + INS & $(6)$ & $90.8 \pm 12.5 \%$ & $86.6 \pm 12.8 \%$ & $103.5 \pm 15 \%$ \\
\hline
\end{tabular}

* Controls denote cultures carried out in SFM containing $2.10^{-11} \mathrm{M}$ CCK-8 and $3.10^{-6} \mathrm{M}$ DXM. Values represent percentage (mean $\pm \mathrm{SD}$ of six experiments) of the control ASA after 2,4 , and 6 days of cultures in fetal pancreas explants exposed to streptozotocin $\left(10^{-7} \mathrm{M}\right)$ during the first $24 \mathrm{~h}$ of culture. Insulin $(0.1 \mathrm{U} / \mathrm{ml})$ was (STZ + INS) or was not (STZ) added to the cultures.

$\dagger$ Indicates significant differences in comparison to controls; $\dagger p<$ 0.05 .

increasing the glucocorticoid binding capacity of the acinar cells $(22,23)$. Herein T3 was used because it is the active hormone. T3 did not enhance the effect of DXM on the ASA in the fetal pancreas cultures (Fig. 2). These results suggest that the mechanisms involved in the T3-DXM interaction later in development are not mature in the fetal pancreas. When used alone in the SFM, T3 quickened the decrease of ASA, as did glucagon (Fig. 1).

An important finding in our experiments was that involving CCK-8. This hormone was previously shown to have trophic effects on the adult rat pancreas in vivo (10) as well as in vitro (17). Recently, Werlin and Stefaniak (5) have shown that injections of CCK-8 in pregnant rats enhanced the ASA in the fullterm pancreas. Unfortunately, the authors could not conclude whether these results were secondary to effect on the mother or direct effects of the hormone on the fetus. In our in vitro study, CCK- 8 by itself significantly reduced the decrease of ASA that is normally observed when fetal pancreas is cultured in a hormonefree medium at a late gestation stage (Fig. $1 B$ ). The effect of CCK-8 was dose dependent, with an optimum between $10^{-12}$ and $10^{-10} \mathrm{M}$ (Fig. 3). This dose-dependent effect in vitro with a significant drop in effect at the highest concentrations matched the results of Werlin and Stefaniak (5) who obtain higher ASA in the term fetal pancreas when the pregnant rats were injected with a CCK-8 dose of $2.5 \mu \mathrm{g} / \mathrm{kg}$ than when they were injected with $10 \mu \mathrm{g} / \mathrm{kg}$. This may indirectly support the interpretation that the CCK-8 effect in vivo is a direct action of the hormone on the fetal pancreas. In our culture system, a combination of CCK-8 and DXM (Fig. $2 B$ ) allowed a nearly complete maintenance of the ASA during the first 4 days of culture. A comparison of the results obtained with the CCK-8 alone (Fig. $1 B$ ) the DXM alone (Fig. $2 A$ ), or the CCK-8-DXM combination (Fig. 2B) shows that the action of both hormones was different, because an additive but not synergistic effect was seen. These results also suggest that CCK-8 is able to act directly on the fetal pancreas. 
an additive but not synergistic effect was seen. These results also suggest that $\mathrm{CCK}-8$ is able to act directly on the fetal pancreas.

How CCK- 8 acts on the fetal acinar cell still remains unclear. Previous studies have suggested that the unresponsiveness of the term fetal rat pancreas to secretagogue stimulation was related to the low binding capacity of the receptors, whereas the underlying machinery of the stimulus-secretion coupling was already mature $(24,25)$. However, it was suggested in a recent report (26), that the fetal and the neonatal rat pancreas had the same binding capacity to CCK -8 . The difference in responsiveness to CCK-8, in terms of amylase release, was viewed as related to an immaturity of the signal transduction events distal to calcium mobilization. In the present study, we have shown that whereas CCK-8 did not stimulate amylase release in the fetal pancreas (data not shown) it already had an effect on the tissue amylase at this age. Furthermore, because the CCK- 8 effect could be inhibited by asperlicin, a potent CCK -8 antagonist that interacts competitively with the CCK receptors (18), our data suggest also that the membrane binding of CCK -8 is already present in the fetus. Further, the pathway leading to the trophic effect that is present at this stage is in contrast to those pathways for the stimulus-secretion coupling process that are immature at the same stage.

The role of insulin on the exocrine pancreas in the rat in vivo has been shown by other authors (11). This hormone is also indispensable in the maintainance of rat adult acinar cells (17), or fetal pancreata (19) for a long time in vitro. Previously we have shown that neonatal streptozotocin-induced diabetes in rat resulted in a transient drop of amylase activity (27). This effect was partially attenuated by insulin supplementation. Inasmuch as insulin was shown to be secreted very early in the fetal rat (28), the possible presence of a functioning $\beta$-cell-acinar-cell axis at this stage was intriguing. Our results (Table 2) showed that after 4 days, the ASA was significantly lower in the fetal pancreata cultured after a 24-h initial exposure to streptozotocin. The fact that insulin can abolish the effect of streptozotocin, which has been shown to damage $\beta$-cells in vitro (29), suggests that this hormone plays a role in the prenatal amylase synthesis by the acinar cells. As reported by Sanders and Rutter (15) with similar doses, insulin in our system (data not shown) did not modulate the enzymatic activities of cultured fetal pancreata. However, in the study by Sanders and Rutter (15), they were not able to show any effect of antiinsulin antiserum in the medium. It can be speculated that the effect of insulin on the acinar cells is a paracine effect that works at its optimal level in the whole pancreas and that antiinsulin antiserum were unable either to bind all the insulin molecules or to mask the active sites of the hormone.

In conclusion, we have shown herein that in addition to glucocorticoids, two other hormones, CCK and insulin, are required to maintain the amylase activity in the fetal exocrine pancreas in vitro. However, before accepting the physiological relevance of the CCK effect, an intestinal secretion of this hormone in the fetal rat, or its possible transfer through the placenta, have to be further demonstrated.

\section{REFERENCES}

1. Ghitens $S 1986$ Differentiation and development of the exocrine pancreas in animals. In: Go VLW, Gardner JD, Brooks FP, Lebenthal E, DiMagno EP, Scheele GA (eds) The Exocrine Pancreas: Biology, Pathology and Disease. Raven Press, New York, pp 21-32
Raven Press, New York, pp 21-32

2. VanAssche FA 1971 Quantitative histology of the pancreas in decapitated and normal rat fetus. Horm Metab Res 3:285-286

3. Deschodt-Lanckman M, Robberecht P, Camus J, Baya C, Christophe J 1984 Hormonal and dietary adaptation of rat pancreatic hydrolases before and after weaning. Am J Physiol 226:39-44

4. Werlin SL, Stefaniak J 1982 Maturation of secretory function in rat pancreas. Pediatr Res 16:123-125

5. Werlin SL, Stefaniak J 1983 Effects of cholecystokinin octapeptide and hydrocortisone on the development of fetal rat pancreas. Bio Neonate 44:287-294

6. Rall L, Pictet R, Ghitens S, Rutter WJ 1977 Glucocorticoids modulate the in vitro development of the embryonic rat pancreas. J Cell Biol 75:398-409

7. Ronzio RA, Rutter WJ 1973 Effects of a partially purified factor from chick embryos on macromolecular synthesis of embryonic pancreatic epithelia. Dev Biol 30:307-320

8. McEvoy RC, Hegre OD, Lazarow A 1976 Fetal and neonatal rat pancreas in organ culture. Age-related effects of corticosterone on the acinar components. Am J Anat 146:133-150

9. Murrell LR, Germain KH, Lynch DM 1975: Survival of functional pancreatic acinar tissue in circumfusion organ culture enhanced by chemically defined medium with hydrocortisone. Cancer Res 35:2286-2288

10. Barrowman JA, Mayston PD 1974: The trophic influence of cholecystokinin on the rat pancreas. J Physiol (Lond) 238:73P-75P

11. Williams JA, Foldfine ID 1986 The insulin-acinar relationship. In: Go LVLW, Gardner J.D, Brooks FP, Lebenthal E, DiMagno EP, Scheele GA (eds). The Exocrine Pancreas: Biology, Pathology and Disease. Raven Press, New York, pp 347-360

12. Kumegawa M, Maeda N, Yajima T, Takuma T, Ikeda E, Hanai H 1980 Permissive role of $\mathrm{L}$-thyroxine in induction of pancreatic amylase by cortisol in neonatal rat. J Endocrinol 86:497-500

13. Brants F, Morisset J 1976 Trophic effect of cholecystokinin-pancreozymin on pancreatic acinar cells from rats of different ages. Proc Soc Exp Biol Med 153:523-527

14. O'Loughlin EV, Chung M, Hollender M, Hayden J, Zahavi I, Gall DG 1985 Effect of epidermal growth factor on ontogeny of the gastrointestinal tract. Am J Physiol 249:G674-G678

15. Sanders TG, Rutter WJ 1974 The developmental regulation of amylolytic and proteolytic enzymes in the embryonic pancreas. J Biol Chem 249:3500-3509

16. Lowry OH, Rosebrough NJ, Farr AL, Randall RJ 1951 Protein measurement with the folin phenol reagent. J Biol Chem 193:265-275

17. Logsdon CD 1986 Stimulation of pancreatic acinar cell growth by CCK, EGF, and insulin in vitro. Am J Physiol 251:G487-G494

18. Chang RSL, Lotti VJ, Monaghan RL, Birbaum J, Stapley EO, Goetz MA, Albers-Schonberg G, Patchett AA, Liesch JM, Hensano OD, Springer JP 1985 A potent nonpeptide cholecystokinin antagonist selective for peripheral tissues isolated from Aspergillus alliaceus. Science 230:177-179

19. Parsa I, Marsh WH 1976 Long-term organ culture of embryonic rat pancreas in a chemically defined medium. Am J Pathol 82:119-125

20. Beaulieu JF, Menard D, Calvert R 1985 Influence of EGF on the maturation of fetal mouse duodenum in organ culture. $\mathrm{J}$ Pediatr Gastroenterol Nutr 4:476-481

21. Leung YK, Elitsur Y, Lee PC, Lebenthal E 1986 Binding of EGF to pancreatic acini of fetal and postnatal rats and possible transfer of EGF through the placenta. Gastroenterology 90:1518(abstr)

22. Lu RB, Chaichanwatanukul K, Lin CH, Lebenthal E, Lee PC 1988 Thyroxine effect on exocrine pancreatic development in rats. Am J Physiol 754: G315G321

23. Lu RB, Lebenthal E, Lee PC 1986 Induction of pancreatic glucocorticoid receptors by thyroxine in rats. Dig Dis Sci 31:1140(abstr)

24. Lebenthal E, Leung YK 1986 Neonatal exocrine pancreatic secretory immaturity: potential mechanisms and investigative approaches. J Pediatr Gastroenterol Nutr 5:1-8

25. Leung YK, Lee PC, Lebenthal E 1986 Maturation of CCK receptors in pancreatic acini of rats. Am J Physiol 250:G594-G597

26. Chang A, Jamieson JD 1986 Stimulus-secretion coupling in the developing exocrine pancreas: Secretory responsiveness to cholecystokinin. J Cell Biol 103:2353-2365

27. Lee PC, Brooks S, Lebenthal E 1986 Streptozotocin effect on the development of the exocrine pancreas in the neonate rats. Diabetes Res Clin Pract 2:269275

28. Hegre OD, McEvoy RC, Bachelder V, Lazarow A 1973 Fetal rat pancreas: differentiation of the islet cell component in vivo and in vitro. Diabetes 22:577-583

29. Bolaffi JL, Nowlain RE, Cruz L, Grodsky GM 1986 Progressive damage of cultured pancreatic islets after single early exposure to streptozotocin. Diabetes $35: 1027-1033$ 